\title{
Häufigkeit von Patienten mit chronischer Dyspnoe in drei deutschen Facharztpraxen
}

\author{
Number of Patients with Chronic Dyspnea in Three German Specialist Practices
}

Autoren

Institute
R. Ewert ${ }^{1}$, C. Bahr ${ }^{2}$, C. Weirich ${ }^{2}$, F. Henschel ${ }^{3}$, A. Rink ${ }^{3}$, J. Winkler ${ }^{4}$

Universitätsmedizin Greifswald, Klinik für Innere Medizin B (Direktor: Prof. Dr. med. S. B. Felix)

${ }^{2}$ Gemeinschaftspraxis Innere Medizin/Kardiologie Pasewalk

${ }^{3}$ Kardiologische Gemeinschaftspraxis Rostock

${ }^{4}$ Praxis für Pneumologie/Allergologie Leipzig $\begin{array}{lll}\text { eingereicht } & \text { 9. 8. } 2012\end{array}$ akzeptiert nach Revision 13. 8.2012

Bibliografie

DOI http://dx.doi.org/

10.1055/s-0032-1325728

Online-Publikation: 25.9.2012

Pneumologie 2012; 66: 662-665

(c) Georg Thieme Verlag KG

Stuttgart · New York

ISSN 0934-8387

Korrespondenzadresse

Prof. Dr. med. Ralf Ewert

Ernst-Moritz-Arndt-Universität

Greifswald, Universitätsmedizin

Klinik für Innere Medizin B,

Bereich Pneumologie/

Infektiologie

Fleischmannstraße 8

17475 Greifswald

ewert@uni-greifswald.de

\section{Zusammenfassung \\ $\nabla$}

Im 2. Quartal 2011 wurde mittels eines selbstentwickelten Fragebogens die Häufigkeit des Symptoms "chronische Dyspnoe" bei Patienten in drei Facharztpraxen ermittelt. Es konnten 1286 anonymisierte Fragebögen mit vollständigen Angaben ausgewertet werden. Der Anteil der Patienten mit einer chronischen Dyspnoe (inklusive „Dyspnoe und Schwächegefühl“) lag in den Praxen zwischen 15,3-57,7 Prozent. Bei alleiniger Betrachtung des Symptoms „chronische Dyspnoe“ zeigte sich, dass diese bei 10,4-31,4\% der Patienten erfasst werden konnte. Aus der Sicht der betreuenden Ärzte und Ärztinnen war die Ursache der chronischen Dyspnoe durch die vorliegenden Informationen ausreichend erklärbar.

\section{Einleitung \\ $\nabla$}

Dyspnoe ist eines der häufigsten Symptome bei Patienten, ungeachtet der vielfältig zugrundeliegenden Störungen und Erkrankungen. Dabei wird die „Dyspnoe als ein subjektives Mißempfinden im Zusammenhang mit der Atmung“ definiert und unterliegt daher in hohem Maße der Persönlichkeit des Patienten. Aktuell wird die Entstehung der Dyspnoe damit erklärt, dass die Stimulation zentraler Areale (über chemische Faktoren, wie $\mathrm{pH}-$ Wert, $\mathrm{CO}_{2}$ - und $\mathrm{O}_{2}$-Sensoren) sowie Mechanorezeptoren aus der Muskulatur sowie den Sehnen und intrapulmonalen Rezeptoren (C-Fasern im Lungenparenchym; J-Fasern in den Bronchien und Gefäßen) nicht zu einer entsprechenden ventilatorischen Antwort führt [13]. Es wurden verschiedene Instrumente zur Erfassung und Quantifizierung der akuten und chronischen Dyspnoe etabliert, wobei das Spektrum von visuellen Analogskalierungen bis hin zur Beschreibung der Dyspnoe im Rahmen von mehrdimensionalen Fragebögen zur Lebensqualität reicht [4-7].

\section{Abstract \\ $\nabla$}

In the second quarter 2011 the incidence of the symptoms "chronic dyspnea" among the patients in three specialist practices was determined by means of a self-developed questionnaire. 1286 anonymised questionnaires with full information could be evaluated. The proportion of the patients with a chronic dyspnea (inclusive "dyspnea and weakness feeling") in the practices amounted to between 15.3 and $57.7 \%$. An exclusive assessment of chronic dyspnea could be assigned to 10.4 to $31.4 \%$ of the patients evaluated. From the view point of the treating physicians, the cause of the chronic dyspnea was sufficiently explained by the available information.

Während eine Reihe von Daten zur Häufigkeit und Differenzierung von Patienten mit akuter Dyspnoe vorrangig aus Notaufnahmen von Krankenhäusern publiziert wurden [8-11], sind solche Informationen zur chronischen Dyspnoe seltener. Für Deutschland liegen Informationen zur chronischen Dyspnoe aus einer Befragung in Sachsen-Anhalt und Schleswig-Holstein in 126 Allgemeinpraxen mit mehr als 4000 Patienten (Alter im Mittel bei 56 Jahren) vor [12]. Dabei bejahte jeder vierte Patient die Frage nach dem Symptom „Atemnot beim Treppensteigen“, und jeder zehnte Patient gab „Luftnot beim Gehen in der Ebene" an. Nächtliche Luftnot wurde von weniger als $10 \%$ der Patienten angeben. In einer populationsbezogenen Studie in Australien (Befragung von 5331 Probanden) gaben 11,1\% chronische Dyspnoe an [13]. Im Rahmen einer populationsbezogenen Studie in Norddeutschland wurden zwischen 1997-2001 insgesamt 4310 Probanden befragt, wobei $17,7 \%$ über eine Luftnot bei Belastung berichteten (unpublizierte Daten). In Studien aus allgemeinärztlichen Praxen [14-15] wird die Dyspnoe als führender 
Datenblatt - Erfassung Dyspnoe

ID-Nummer:

Männlich $\square \quad$ Weiblich $\square \quad$ Körpergröße:... cm Körpergewicht:... kg

1 Haben Sie bei körperlicher Belastung Luftnot oder Schwächegefühl oder beides? Nur Luftnot

Nur Schwächegefühl

Beides

keine Beschwerden

2 Haben Sie gelegentlich auch in Ruhe Luftnot oder wachen Sie nachts wegen Luftnot auf Nein

Ja

3 Haben Sie am Abend gelegentlich geschwollene Beine? Nein

4 Müssen Sie nachts häufig zum Wasserlassen aufstehen? Nein

Ja

5 Ist bei Ihnen eine chronische Lungen (z.B. Asthma, COPD)- oder Herzerkrankung (Herzschwäche, Rhythmusstörungen) bekannt? Nein

Ja

6 Sind bei Ihnen andere Erkrankungen bekannt (Lungenembolie, Bindegewebs-, Blut- oder Lebererkrankungen, Bluthochdruck, Blutzuckererkrankung/Diabetes) Nein

Ja

7 Ist bei Ihnen eine Allergie (oder „Heuschnupfen“) ärztlich festgestellt worden? Nein

Ja

8 Sind Sie Raucher bzw. waren Sie Raucher?

Ja

Nein
Abb. 1 Fragebogen.

\section{Methodik}

In zwei Facharztpraxen für Kardiologie $(1 \times$ Stadt mit $>200000$ Einwohnern [Praxis 1]; $1 \times$ Stadt mit 11000 Einwohnern und ländlichem Umfeld [Praxis 2]) und in einer Facharztpraxis für Pneumologie (Stadt mit > 500000 Einwohnern [Praxis 3]) wurden im II. Quartal 2011 Patienten zur Teilnahme an einer anonymisierten Befragung zum Vorliegen einer chronischen Dyspnoe eingeladen. Nach Aufklärung über die Zielstellung der geplanten Befragung mit nachfolgender Datenerhebung wurde das Einverständnis der Patienten dokumentiert.
Vom Praxispersonal wurde den Patienten ein speziell entwickelter Fragebogen ausgehändigt, welcher das Vorliegen einer chronischen Dyspnoe (d. h. länger als zwei Wochen bestehend) erfasste. Wenn dieser Sachverhalt positiv beantwortet wurde, sind weitere Fragen zu ausgewählten Symptomen/Beschwerden und zum Rauchverhalten gestellt worden ( $\bullet$ Abb.1). In dem vom Arzt auszufüllenden Teil wurden zwei Fragen („Sind Vorbefunde bekannt?“ bzw. „Sind auffällige Laborbefunde vorhanden?“) gestellt, und anschließend sollte ein Gesamturteil („Ist die Dyspnoe ausreichend durch die vorliegenden Informationen erklärt?“) abgegeben werden.

Die Patienten wurden konsekutiv zur Teilnahme an der Befragung eingeladen, wobei Patienten mit alleiniger Vorstellung zum Erhalt eines Rezeptes, zur Teilnahme an einer Patientenschulung, zur Durchführung geplanter Prozeduren (Desensibilisierung, Schrittmacherabfrage, Anlegen eines Langzeit-EKGs oder einer 24-Stunden-Blutdruck-Messung, Karotis-Duplex-Sonografie u.a.m.) nicht berücksichtigt wurden. 


\begin{tabular}{|c|c|c|c|}
\hline Fragestellung & Praxis 1 & Praxis 2 & Praxis 3 \\
\hline $\begin{array}{l}\text { „Haben Sie gelegentlich auch in Ruhe Luftnot oder wachen Sie nachts wegen } \\
\text { Luftnot auf?" }\end{array}$ & 39,7 & 16,6 & 30,5 \\
\hline „Haben Sie am Abend gelegentlich geschwollene Beine?“ & 40,3 & 48,1 & 49,0 \\
\hline „Müssen Sie nachts häufig zum Wasserlassen aufstehen?“ & 45,8 & 46,3 & 51,9 \\
\hline „Ist bei Ihnen eine chronische Lungen- oder Herzerkrankung bekannt?“ & 54,2 & 37,0 & 83,9 \\
\hline „Sind bei Ihnen andere Erkrankungen bekannt?" & 81,9 & 48,1 & 68,0 \\
\hline „Ist bei Ihnen eine Allergie (oder „Heuschnupfen“) ärztlich festgestellt worden?“ & 16,7 & 9,3 & 40,7 \\
\hline „Sind Sie Raucher bzw. waren Sie Raucher?" & 19,4 & 24,1 & 41,9 \\
\hline
\end{tabular}

Tab. 1 Ergebnisse der Befragung bei Patienten (Anteil positiver Antworten in Prozent).

\section{Datenerfassung}

$\nabla$

Die Ergebnisse der Fragebögen wurden in einer webbasierten, anonymisierten Datenbank erfasst und ausgewertet (mit Unterstützung der Fa. Docxcellence GmbH, Berlin).

Zur Durchführung der Befragung existiert ein Votum der Ethikkommission der Ernst-Moritz-Arndt Universität Greifswald vom 22.02.2011 (Reg.-Nr.03/10a).

\section{Ergebnisse \\ $\nabla$}

Zur Auswertung standen 1286 Fragebögen mit vollständigen Angaben zur Verfügung. Drei Bögen wurden aufgrund unvollständiger Angaben von der Auswertung ausgeschlossen. Es wurde in den Praxen nach Beginn der Studie deutlich, dass ein Teil der Patienten nicht selbstständig zur Beantwortung der Fragen in der Lage war. Etwa ein Drittel der Patienten benötigte Unterstützung zum Ausfüllen der Fragebögen durch das Praxispersonal. Aufgrund mangelnder personeller Ressourcen wurde die Befragung in der Praxis 2 (Kardiologie) vorzeitig eingestellt, jedoch wurden die Daten in die Analyse einbezogen.

Das Geschlechterverhältnis der teilnehmenden Personen war weitgehend ausgeglichen, wobei in 73 Fällen die Angaben zum Geschlecht fehlten. Die Angaben zum Körpergewicht und der Körpergröße waren häufig unvollständig, sodass keine Korrektur der Daten hinsichtlich des BMI möglich war.

Der Anteil der Patienten mit einer chronischen Dyspnoe (inklusive „Dyspnoe und Schwächegefühl“) lag in der Praxis 1 (Kardiologie) bei 15,3\%; in der Praxis 2 (Kardiologie) bei 50,9\% und in der Praxis 3 (Pneumologie) bei 57,7\%. Ausschließlich eine „chronische Dyspnoe“ lag bei 10,4\%, 39,6\% bzw. 31,4\% der Patienten $\operatorname{vor}(\bullet$ Abb.2).

In Tab. 1 wurde der prozentuale Anteil der von den Patienten mit einer chronischen Dyspnoe (inklusive „Dyspnoe und Schwächegefühl“) positiv beantworteten Fragen dargestellt.

Von den Ärzten und Ärztinnen wurde angegeben, dass bei 91,6\% (Praxis 1), 83,3\% (Praxis 2) und 24,9\% (Praxis 3) der Patienten Vorbefunde bekannt waren. Auffällige Laborbefunde lagen nur bei 9,7\% (Praxis 1), bei 3,7\% (Praxis 2) und 8,8\% (Praxis 3) der Patienten vor.

Im Ergebniss mussten die Ärzte und Ärztinnen ein Urteil zur Dyspnoe („Ist die Dyspnoe ausreichend durch die vorliegenden Informationen erklärt?“) abgeben, wobei in der Praxis 1 diese Frage bei $60 \%$ (43/72, d.h. 59,7\%), in der Praxis 2 bei $91 \%$ (49/54, d.h. $90,7 \%)$ und in der Praxis 3 bei 78\% (322/410, d.h. 78,5\%) der Patienten positiv beantwortet wurde $(\mathbb{A b b} \mathbf{\text { Ab }})$.

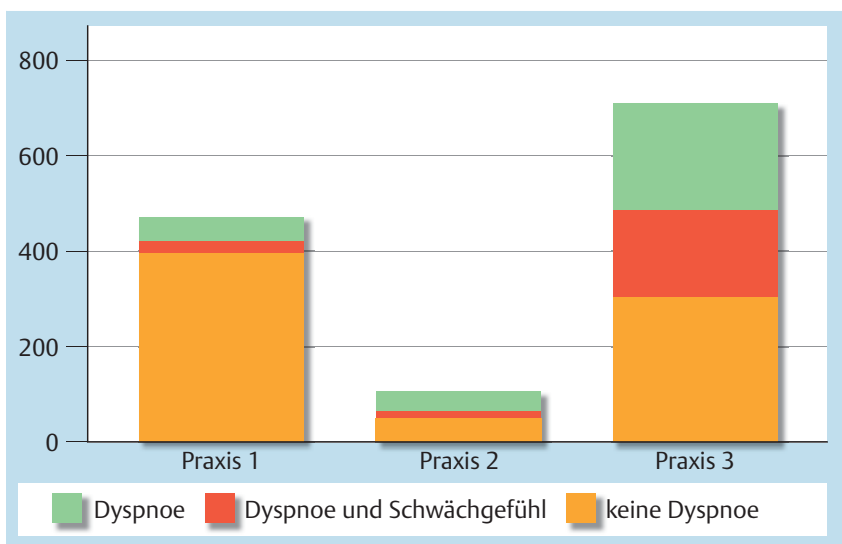

Abb.2 Umfang und Verteilung der Patienten in den drei Praxen.

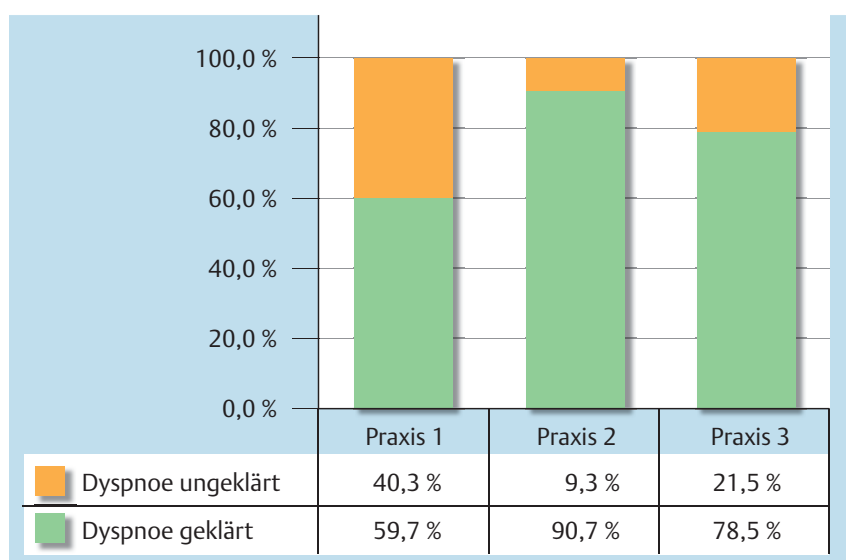

Abb.3 Anteil der Patienten mit ausreichend geklärter Ursache der Dyspnoe.

\section{Diskussion}

Der Anteil der Patienten mit einer chronischen Dyspnoe (inklusive „Dyspnoe und Schwächegefühl“) wurde mithilfe eines Fragebogens in kardiologischen Facharztpraxen mit 15,3\% bzw. 50,9\% und in einer pneumologischen Facharztpraxis mit 57,7\% der befragten Patienten ermittelt. Ausschließlich eine chronische Dyspnoe wurde von 10,4\%, 39,6\% bzw. 31,4\% der befragten Patienten angegeben. Die Unterschiede des prozentualen Anteils der Patienten mit einer chronischen Dyspnoe sind augenfällig, können jedoch nicht mit den zur Verfügung stehenden Daten (Datenumfang, Art der Erhebung) erklärt werden. Als mögliche Ursache kann eine unterschiedliche Patientenstruktur bzw. Überweisungsverhalten in den beiden kardiologischen Praxen gelten, wobei diese Frage nicht Gegenstand der Auswertung war. Über alle drei Praxen gesehen, gaben im Mittel 24,4\% aller befragten Pa- 
tienten eine alleinige chronische Dyspnoe an. Dieser Anteil von Patienten mit chronischer Dyspnoe wurde auch bei einer Befragung von mehr als 4000 Patienten in Allgemeinpraxen dokumentiert [12].

Auffällig bei der Auswertung der Daten war ein sehr unterschiedlicher Anteil der Patienten mit bekannten Vorbefunden, wobei dieser in den kardiologischen Praxen bei 80-90\% und in der pneumologischen Praxis lediglich bei $25 \%$ der Patienten angegeben wurde. Die Ursachen für diesen Unterschied zwischen den Praxen in unserer Untersuchung bleiben offen.

Die von uns durchgeführte Befragung zeigt, dass eine von den Patienten mitgeteilte chronische Dyspnoe (inklusive „Dyspnoe und Schwächegefühl“) mittels des verwendeten Fragebogens und bei Kenntnis von Vorbefunden, welche durch die Patienten mitgebracht wurden, in 60-90\% der Fälle als ausreichend geklärt angesehen werden kann. Die Mittelung der Ergebnisse unserer Befragung über die drei Praxen macht, ungeachtet der methodischen Limitierungen eines solchen Vorgehens, deutlich, dass bei etwa 75\% (414/536) aller Patienten mit chronischer Dyspnoe (inklusive „Dyspnoe und Schwächegefühl“) die Ursache als ausreichend geklärt angesehen wird.

Somit kann festgestellt werden, dass bei nur 25\% der Patienten mit chronischer Dyspnoe weiterführende Untersuchungen zur ausreichenden Klärung der Ursache notwendig sind.

Die Limitierungen der vorliegenden Studie sind u.a. in der fehlenden Vollerfassung der Patienten in den untersuchten Praxen zu suchen. Die Erhebung eines (wenn auch kurzen) Fragebogens in einer Facharztpraxis ist mit einer relevanten zusätzlichen Belastung für das Praxispersonal verbunden. In unserer Erhebung wurde deutlich, dass etwa ein Drittel der Patienten Unterstützung beim Ausfüllen des Fragebogens benötigte. Daher sollten bei zukünftigen Untersuchungen mit Fragebögen zusätzliche personelle Ressourcen eingeplant werden. Der von uns verwendete Fragebogen wurde bisher nicht zur Erhebung einer chronischen Dyspnoe in der Praxis validiert. Dieses stellt jedoch ein generelles Problem der meisten verwendeten Fragebögen dar, welche überwiegend zur Erfassung der Dyspnoe bei bestimmten Erkrankungen oder für epidemiologische Studien validiert wurden [16].

\section{Danksagung}

Das Projekt wurde mit finanzieller Unterstützung durch die Firma Actelion Pharmaceuticals Deutschland GmbH durchgeführt.

\section{Interessenkonflikt}

$\nabla$

R. Ewert und J. Winkler haben Erstattungen von Reisekosten und Teilnahmegebühren für Kongresse, Honorare für Vorträge und finanzielle Zuwendungen für die Teilnahme an klinischen Studien erhalten. C. Bahr, C. Weirich, F. Henschel und A. Rink geben an, dass kein Interessenkonflikt besteht.

\section{Literatur}

1 Burki NK, Lee LY. Mechanisms of Dyspnea. Chest 2010; 138: 1196 - 1201

2 Nishino T. Dyspnoea: underlying mechanisms and treatment. Brit J Anaesthesia 2011; 106: $463-474$

3 Parshall MB, Schwartzstein RM, Adams $L$ et al. An Official American Thoracic Society Statement: Update on the Mechanisms, Assessment, and Management of Dyspnea. Am J Respir Crit Care Med 2012; 185 : $435-452$

4 Ewert R, Winkler J, Opitz C. Luftnot: Erfassung, Graduierung und Differenzierung. Atemwegs- und Lungenkrankheiten 2012; 38: 128-136

5 Eakin EG, Sassi-Dambron DE, Ries $A L$ et al. Reliability and validity of dyspnoea measures in patients with obstructive lung diseases. Intern J Behavioral Medicine 1995; 2: 118 -134

6 Cullen DL, Rodak B. Clinical utility of measures of breathlessness. Respir Care 2002; 47: 986-993

7 Garin 0 , Ferrer M, Pont A et al. Disease-specific health-related quality of life questionnaires for heart failure: a systematic review with meta-analysis. Qual Life Res 2009; 18: 71 - 85

8 Brown LH, Gough JE, Seim RH. Can quantitative capnometry differentiate between cardiac and obstructive causes of respiratory distress? Chest 1998; 113: $323-326$

9 McNamara RM, Cionni DJ. Utility of the peak expiratory flow rate in the differentiation of acute dyspnea. Cardiac vs pulmonary origin. Chest 1992; 101: 129-132

10 Gough JE, Brewer KL. Can peak expiratory flow measurements differentiate chronic obstructive pulmonary disease from congestive heart failure? Emerg Med Int 2012: Epub 2011 Oct 27

11 Ailani RK, Ravakhah K, DiGiovine B et al. Dyspnea differentiation index: A new method for the rapid separation of cardiac vs pulmonary dyspnea. Chest 1999; 116: 1100-1104

12 Magnussen H. Prävalenz respiratorischer Symptome - Aktuelle Datenlage in deutschen Praxen. Med. Klinik 2005; 100 : 1 -4

13 Bowden JA, To TH, Abernethy AP et al. Predictors of chronic breathlessness: a large population study. BMC Public Health 2011; 11: 33

14 Frese T, Sobeck C, Herrmann K et al. Dyspnea as the reason for encounter in general practice. J Clin Med Res 2011; 3: 239-246

15 Charles J, $\mathrm{Ng} \mathrm{A}$, Britt $\mathrm{H}$. Presentations of shortness of breath in Australian general practice. Aust Fam Physician 2005; 34: 520-521

16 Mahler DA. How should health-related quality of life be assessed in patients with COPD? Chest 2000; 117 (Suppl. 02): 54S-57S 